\title{
The emergent relationship between solvent-free chemistry, crystal engineering and mineralogy
}

Tomislav Friščić, Igor Huskić

Department of Chemistry, McGill University, 801 Sherbrooke St. W. H3A 0B8 Montreal, Canada. tomislav.friscic@mcgill.ca

The structural characterization and discovery of new minerals with metal-organic framework (MOF) structures (Fig. 1) has challenged the view of MOFs as purely synthetic materials.[1] Almost simultaneously, the increasing popularity of solvent-free synthetic techniques, including mechanochemistry and geochemically-inspired "accelerated aging"[2] has enabled a cleaner, more environmentally-friendly synthesis of MOFs from mineral-like feedstocks. These recent developments highlight the previously unknown relationships between geology and MOF chemistry.[3]

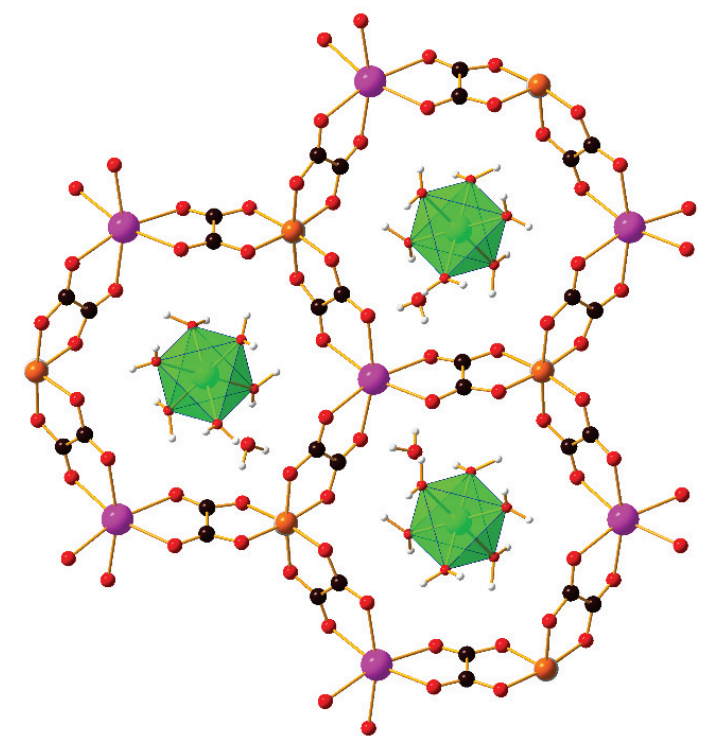

Fig. 1. Fragment of the structure of stepanovite, a naturally-ocurring MOF mineral.[1]

This presentation will outline the selected examples of MOF structures found in minerals, noting their functional properties such as proton conductivity.[4] At the same time, we will also describe the emergent geologically-inspired approaches to MOF synthesis, as a means to highlight how the emergent application of geomimetic concepts in MOF chemistry can lead to advances in the design and synthesis of MOFs.

\section{$\underline{\text { References }}$}

[1] Huskić, I. et al. (2016) Science Adv., 2, e1600621

[2] Mottillo, C. \& Friščić, T. (2017) Molecules, 22, 144.

[3] Huskić, I. \& Friščić, T. (2018) Acta Crystallogr. B, 74, 539-559.

[4] Huskić, I. et al. (2019) Chem. Sci., doi:10.1039/C8SC05088K 